\title{
In focus in Bad Ischl: Golgi apparatus 2013
}

\author{
Margit Pavelka $\cdot$ Jürgen Roth
}

Accepted: 2 July 2013/Published online: 24 July 2013

(c) Springer-Verlag Berlin Heidelberg 2013

From September 17 to 19, 2013, Bad Ischl in the Salzkammergut, Austria will host an international symposium dedicated to a highly complex organelle, the Golgi apparatus. Although 115 years have passed since its first description by Camillo Golgi in 1898 (Dröscher 1998), the Golgi apparatus is steadily in the focus of interest and subject of continuing scientific controversies. Because of its extraordinarily complex organization and involvement in diverse cellular functions (Fig. 1), the Golgi apparatus still presents a formidable challenge to cell and molecular biologists. Experts from all parts of the world working in different areas of Golgi apparatus research and using different experimental models and

M. Pavelka ( $\square)$

Department of Cell Biology and Ultrastructure Research, Center for Anatomy and Cell Biology,

Medical University of Vienna, 1090 Vienna, Austria

e-mail: margit.pavelka@meduniwien.ac.at

J. Roth

Department of Integrated OMICS for Biomedical Science, WCU Program of Graduate School, Yonsei University,

Seoul 120-749, South Korea

J. Roth

University of Zurich, 8091 Zurich, Switzerland techniques will meet in Bad Ischl to present and discuss recent advances related to the structure and function of this complex organelle.

Lecture sessions and poster presentations are dedicated to key topics of present-day Golgi apparatus research: "The complexity of Golgi trafficking routes in secretion and endocytosis", "Protein dynamics, sorting and recycling", "Models for antero- and retrograde transport across the stacks of cisternae", "Regulation of the Golgi apparatus architecture", "Signalling circuits", "Mechanisms of Golgi cisternal stacking", "Golgi structure-function relationships", "Dynamics at the ER-Golgi-interface and the trans Golgi network", "Molecular machineries and formation of transport carriers", "Golgi apparatus and cytoskeleton", "Golgi-derived microtubules", "The role of the Golgi apparatus in cell polarity and directional migration", "Unconventional transport", "Golgi biogenesis", "The Golgi apparatus in mitosis and cell division", "Golgi organization in response to physiologic and pathologic cellular changes", "Golgi dissociation and reorganization, disassembly, reassembly and new formation", "The Golgi apparatus in plants, algae and yeast", "New technical approaches and novel microscopic methods" (Boncompain and Perez 2013; Chia et al. 2013; Day et al. 2013; Egea et al. 2013; Machamer 2013; Martínez-Alonso et al. 2013; Polishchuk and Lutsenko 2013; Sandvig et al. 2013; Tillmann et al. 2013; Uemura and Nakano 2013; Warren 2013; Willett et al. 2013; Zhu and Kaverina 2013).

The Golgi apparatus as schematically depicted in Fig. 1 serves to demonstrate the organelle's complex and variable appearances and its involvement in diverse cellular activities. It is a main goal of the symposium to unravel the complex relationships between the different structural appearances and the multiple functions and processes in which the Golgi apparatus is involved. 


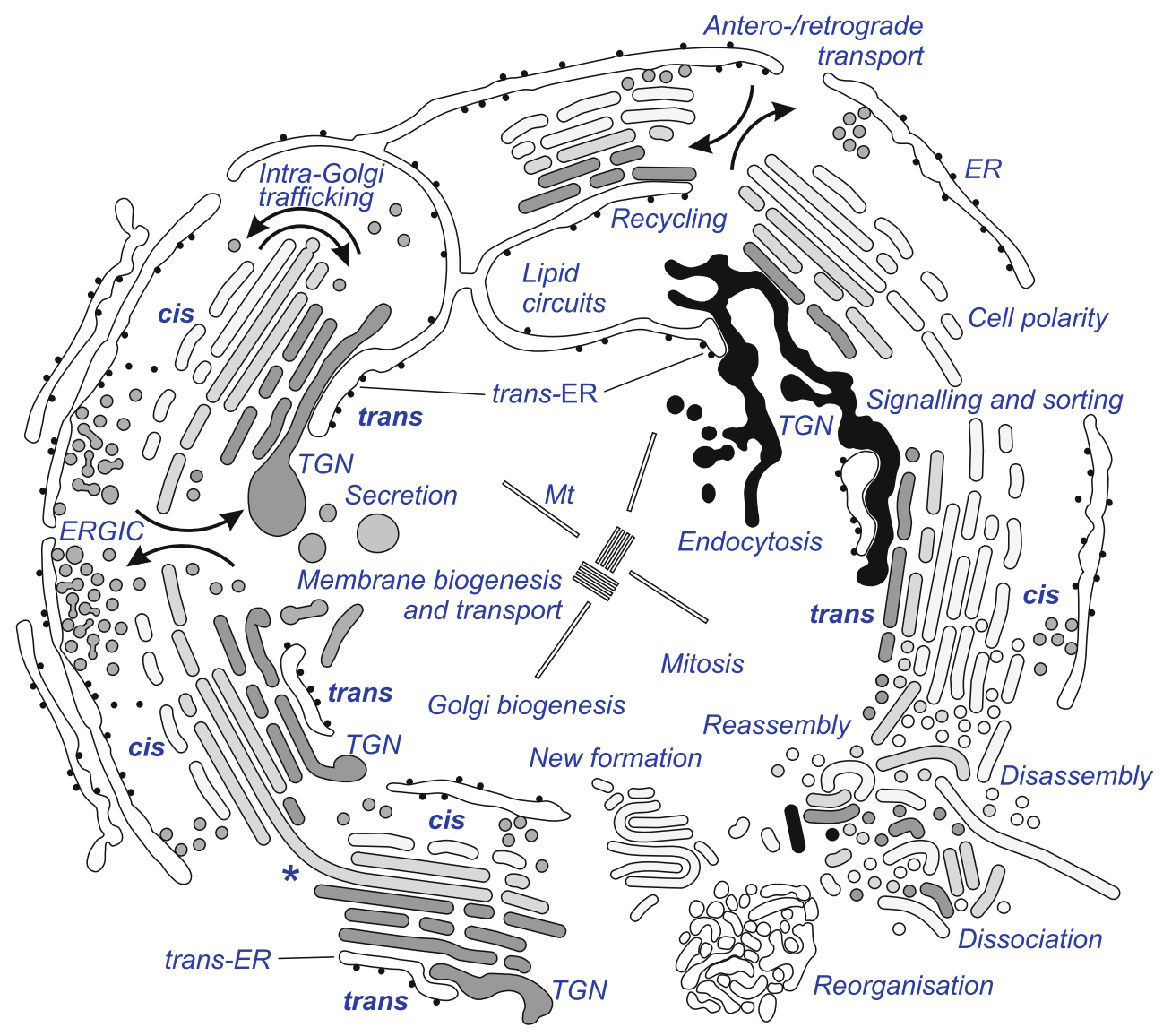

Fig. 1 The interconnected stacks of cisternae with cis-to-trans polarity are composed of flexible cis, medial and trans subcompartments (indicated by increasing staining intensities). The endoplasmic reticulum-Golgi intermediate compartment (ERGIC), trans-Golgi network (TGN) and trans-Golgi-ER help to define the cis and trans sides of the stacks, respectively. However, their presence and dimension depend on the specialization and functional state of cells (shown for secretion on the left-hand side and for endocytosis on the

Acknowledgments The authors like to cordially thank Mr. Thomas Nardelli for his help with the artwork.

\section{References}

Boncompain G, Perez F (2013) The many routes of Golgi-dependent trafficking. Histochem Cell Biol 140:251-260

Chia PZC, Gunn P, Gleeson PA (2013) Cargo trafficking between endosomes and the trans-Golgi network. Histochem Cell Biol 140:307-315

Day KJ, Staehelin LA, Glick BJ (2013) A three-stage model of Golgi structure and function. Histochem Cell Biol 140:339-349

Dröscher A (1998) Camillo Golgi and the discovery of the Golgi apparatus. Histochem Cell Biol 109:425-430

Egea G, Serra-Peinado C, Salcedo-Sicilia L, Gutierrez-Martinez E (2013) Actin acting at the Golgi. Histochem Cell Biol 140:347-360

Machamer CE (2013) Accommodation of large cargo within Golgi cisternae. Histochem Cell Biol 140:261-269 right-hand side; the endocytic TGN is drawn in black). In the middle upper stack, a TGN is missing but trans-Golgi ER is closely attached to the trans cisternae. The left lower part of the drawing shows a "backbone" cisterna (asterisk) connecting stacks with exchanged polarities. The drawings at the right lower section address processes of disassembly and reassembly, dissociation and reorganization of Golgi membranes and new formation of Golgi stacks, as they occur during the cell cycle and in response to drug treatments
Martínez-Alonso E, Tomás M, Martínez-Menárguez JA (2013) Golgi tubules: their structure, formation and role in intra-Golgi transport. Histochem Cell Biol 140:327-339

Polishchuk R, Lutsenko S (2013) Golgi in copper homeostasis: a view from the membrane trafficking field. Histochem Cell Biol 140:285-295

Sandvig K, Skotland T, van Deurs B, Klokk TI (2013) Retrograde transport of protein toxins through the Golgi apparatus. Histochem Cell Biol 140:317-326

Tillmann KD, Millarte V, Farhan H (2013) Regulation of traffic and organelle architecture of the ER-Golgi interface by signal transduction. Histochem Cell Biol 140:297-306

Uemura T, Nakano A (2013) Plant TGNs: dynamics and physiological functions. Histochem Cell Biol 140:341-345

Warren G (2013) Transport through the Golgi in Trypanosoma brucei. Histochem Cell Biol 140:235-238

Willett R, Ungar D, Lupashin V (2013) The Golgi puppet masterCOG complex at center stage of membrane trafficking interactions. Histochem Cell Biol 140:271-283

Zhu X, Kaverina I (2013) Golgi as an MTOC: making microtubules for its own good. Histochem Cell Biol 140:361-367 